\begin{tabular}{|l|}
\hline A\&A manuscript no. \\
(will be inserted by hand later) \\
\hline Your thesaurus codes are: \\
$(\mathbf{0 9 . 1 1 . 1 ; ~ 1 9 . 5 0 . 1 ; 2 4 . 0 2 . 1 )}$ \\
\hline
\end{tabular}

ASTRONOMY AND ASTROPHYSICS 6.1 .2018

\title{
Search for Old Neutron Stars in molecular clouds: Cygnus Rift and Cygnus OB7
}

\author{
T. Belloni ${ }^{1}$, L. Zampieri ${ }^{2}$ 甘, and S. Campana ${ }^{3}$ \\ 1 Astronomical Institute "Anton Pannekoek", University of Amsterdam and Center for High-Energy Astrophysics, Kruislaan \\ 403, NL-1098 SJ Amsterdam, The Netherlands \\ ${ }^{2}$ International School for Advances Studies (S.I.S.S.A.), Via Beirut 2-4, I-34013 Trieste, Italy \\ ${ }^{3}$ Osservatorio Astronomico di Brera, Via Bianchi 46, I-22055 Merate, Italy
}

Received ...; accepted ...

\begin{abstract}
We present the results of a systematic search for old isolated neutron stars (ONSs) in the direction of two giant molecular clouds in Cygnus (Rift and OB7). From theoretical calculations, we expect the detection of a large number of ONSs with the PSPC on board ROSAT. By analyzing the PSPC pointings in the direction of the clouds, we find four sources characterized by count rates $\left(\sim 10^{-3} \mathrm{c} \mathrm{s}^{-1}\right)$ and spectral properties consistent with the hypothesis that the $\mathrm{X}$-ray radiation is produced by ONSs and also characterized by the absence of any measurable optical counterpart within their error circle in the digitized red plates of the Palomar All Sky Survey. The importance of follow-up deep observations in the direction of these ONS candidates is discussed. The observational and theoretical approach presented here could be fruitfully applied also to the systematic search for ONSs in other regions of the Galaxy.
\end{abstract}

Key words: neutron: stars - X-rays: stars - ISM: individual: Cyg OB7 \& Cyg Rift

\section{Introduction}

In the first phases following their formation, neutron stars are probably strongly magnetized and can emit intense electromagnetic dipole radiation at the expense of their rotational energy. These energy losses will produce a nonnegligible radiation pressure on the surrounding medium, inhibiting any possible accretion of interstellar material (see e.g. Lipunov 1992). After the radiation pressure has

Send offprint requests to: T. Belloni

* Present address: University of Illinois at UrbanaChampaign, Department of Physics, Loomis Laboratory of Physics, 1110 West Green Street, Urbana, Illinois 61801-3080, U.S.A. dropped, the flow penetrates inside the accretion radius, proceeding unaffected until the magnetospheric (Alfvèn) radius is reached, where the magnetic pressure becomes equal to the ram pressure of the infalling gas. At this point accretion can continue if the centrifugal acceleration exerted on the matter flowing along the field lines is smaller than the gravitational acceleration: in order to have accretion from the Interstellar Medium (ISM) a relic magnetic field $B \lesssim 10^{9} \mathrm{G}$ and a rotational period greater than a few seconds are needed (Illarionov \& Sunyaev 1975), which are not implausible for Old Neutron Stars (ONSs). If this condition is satisfied, ONSs should appear as weak, soft $\mathrm{X}$-ray sources, as firstly suggested by Ostriker, Rees \& Silk (1970).

The detection of ONSs is a long-sought goal of X-ray astronomy such that it was included as a possible target for the Einstein mission (Helfand, Chanan \& Novick 1980), but the first systematic study of the observability of these sources with ROSAT was carried out by Treves \& Colpi (1991), who found that, in the most favourable case of polar cap accretion, thousands of ONSs should appear in the ROSAT PSPC All Sky Survey. A complete analysis by Blaes \& Madau (1993) essentially confirmed the results of Treves \& Colpi (1991). Zane et al. (1995a) reconsidered the detectability of ONSs with ROSAT and found that, for polar cap accretion, ONSs may contribute up to $10 \%$ of the unresolved X-ray background, although in this case about 10 sources $\mathrm{deg}^{-2}$ should be observable by ROSAT at the sensitivity limit of $10^{-3} \mathrm{c} \mathrm{s}^{-1}$. Finally, in two recent investigations Shemi (1995) and Zane et al. (1995b) have studied the detectability of nearby ONSs. To date, good evidence for ONS candidates has been presented by Stocke et al. (1995) and Walter, Wolk \& Neuhäuser (1996), who found that two unidentified X-ray sources detected in either the Einstein Extended Medium Sensitivity Survey or in the ROSAT All Sky Survey may actually be powered by an accreting ONS. 
A different approach for the search of ONSs was suggested by Blaes \& Madau (1993) and Colpi, Campana \& Treves (1993), who indicate as a very promising environment for their detection nearby giant molecular clouds, where the density of the ISM is sufficiently high for a single neutron star to emit a considerable amount of radiation. However, in this case also the absorption of the ISM will be enhanced and the detectability will crucially rely on the delicate balance between the emitted luminosity and the absorbed flux. As shown by Blaes \& Madau (1993), Colpi, Campana \& Treves (1993) and Zane et al. (1995a), some molecular clouds in the vicinity of the Sun are expected to contain a large number of ONSs emitting at relatively high rates and then representing the most favourable sites for the observability of these sources.

On the wake of these studies, we have performed a systematic investigation of the X-ray sources detected by ROSAT PSPC in the direction of two molecular clouds (Cygnus Rift and Cygnus OB7), which should be particularly favourable as far as the expected number of ONSs is concerned. As we will see, the definition of a systematic searching strategy is very important when dealing with large number of sources and, to this end, the theoretical investigation of the expected emission properties turns out to be certainly a key ingredient.

Section 2 is devoted to the theoretical analysis of the number of ONSs detectable by ROSAT PSPC in the direction of these two molecular clouds and their emission properties, whereas in section 3 we will present the searching procedure and the four ONS candidates which have been found in this way. Finally, section 4 contains a discussion of these results in connection with the detectability issue of ONSs and a suggestion for possible new deep observations to shed further light on the nature of these objects.

\section{Theoretical expectations}

\subsection{Assumptions}

For a star moving supersonically with velocity $v$ relative to the interstellar gas, the amount of mass which is accreted per unit time is $\dot{M} \simeq \pi r_{g c}^{2} n m_{p} v$, where $r_{g c}=$ $2 \chi G M_{*} / v^{2}$ is the gravitational capture radius, $M_{*}$ is the mass of the neutron star, $n$ the number density of the ISM ( $n=1.36 n_{H}$, where $n_{H}$ is the hydrogen number density, for standard chemical composition) and $\chi$ is parameter of order unity which encompasses the theoretical uncertainties on the value of $r_{g c}$. In the following we take $\chi=1$ (see e.g. Novikov \& Thorne 1973). At the typical values of the ISM density (outside molecular clouds), it is not clear that the fluid approximation applies at the gravitational capture radius (see Koester 1976) and the accretion rate could be greatly reduced. However, low magnetic fields, frozen in the interstellar medium, may effectively couple the gas particles. In this case, if the Larmor radius is smaller than the gravitational capture radius, the fluid approximation can be still considered valid and the accretion rate is reasonably expressed through the previous formula. The total luminosity $L$ emitted by an ONS is then

$$
\begin{aligned}
L= & \eta \dot{M} c^{2}=9.5 \times 10^{29}\left(\frac{\eta}{0.18}\right) \\
& \left(\frac{M_{*}}{M_{\odot}}\right)^{2}\left(\frac{n}{1 \mathrm{~cm}^{-3}}\right)\left(\frac{v}{40 \mathrm{~km} \mathrm{~s}^{-1}}\right)^{-3} \mathrm{erg} \mathrm{s}^{-1}
\end{aligned}
$$

where $\eta$ is the relativistic efficiency $(\eta \simeq 0.18$ for a neutron star radius of $r_{*} \simeq 12 \mathrm{~km}$ and a mass of $M_{*} \simeq 1.4 M_{\odot}$ ) and $c$ is the light velocity. If the star moves subsonically relative to the ISM, equation (1) remains valid provided that the ISM sound speed $v_{s}$ is used in place of $v\left(v_{s} \simeq\right.$ $\left.10 \sqrt{\left(T / 10^{4} \mathrm{~K}\right)} \mathrm{km} \mathrm{s}^{-1}\right)$.

As shown by equation (1), in order to investigate the detectability of ONSs, it is crucial to have knowledge of the density distribution of the ISM and the velocity distribution of ONSs in the Galaxy. Calculations of the temporal evolution of the ONS distribution function have been carried out by many authors (Paczyński 1990; Hartmann, Epstein \& Woosley 1990; Blaes \& Rajagopal 1991; Blaes \& Madau 1993; Zane et al. 1995a). Since we are interested to investigate the detectability of ONSs in the solar neighbourhood and close to the Galactic plane, in the following we will use the analytic approximation to the ONS distribution computed by Zane et al. (1995a).

The structure of the Local Interstellar Medium has been widely investigated: within $\sim 50-100 \mathrm{pc}$ from the Sun, the gas is tenuous ( $\left.n \simeq 0.05-0.07 \mathrm{~cm}^{-3}\right)$ and warm $\left(T \simeq 10^{4} \mathrm{~K}\right)$, although the presence of a number of relatively high density regions $\left(n \sim 1 \mathrm{~cm}^{-3}\right)$ can rise the observed column density up to $\sim 2 \times 10^{20} \mathrm{~cm}^{-2}$ along certain line of sights, such as those in the direction of Cygnus Rift and Cygnus OB7 (Frisch and York 1983; Paresce 1984; Welsh et al. 1994; Diamond et al. 1995). On much larger scales, according to Dickey \& Lockman (1990), the average density in the Galactic plane is $n \simeq 1 \mathrm{~cm}^{-3}$ with a scale height variable with the distance from the Sun, although there are a number of tenuous $\left(n \simeq 0.01 \mathrm{~cm}^{-3}\right)$ hot $\left(T \simeq 10^{5}-10^{6} \mathrm{~K}\right)$ bubbles. Since Cygnus Rift and Cygnus OB7 are very close to the Galactic plane $\left(-5^{\circ}<b<5^{\circ}\right)$, in the following the ISM will be approximately described as a piecewise constant density medium with $n_{H}=0.645$ $\mathrm{cm}^{-3}$ for $d<100 \mathrm{pc}$ and $n_{H}=1 \mathrm{~cm}^{-3}$ elsewhere. For the sources embedded in the clouds and the background sources, the contribution of the cloud material to the total absorption will be added.

A thorough investigation of the emitted spectrum turns out to be a further key ingredient in order to correctly address the issue of detecting ONSs, since the choice of the energy bands where to look for and the absorption of the interstellar medium are strongly related to the ONSs emission properties. As shown by Alme \& Wilson (1973), if binary Coulomb collisions between the infalling ions and the atmospheric electrons dominate, as it is expected at 
Table 1. Characteristic parameters for Cygnus Rift and Cygnus OB7

\begin{tabular}{lccccc} 
Cloud & $\begin{array}{c}\text { volume } V_{c} \\
\left(\mathrm{pc}^{3}\right)\end{array}$ & $\begin{array}{c}\text { apparent surface } \Sigma_{c} \\
\left(\mathrm{deg}^{2}\right)\end{array}$ & $\begin{array}{c}\text { inner radius } r_{i n} \\
(\mathrm{pc})\end{array}$ & $\begin{array}{c}\text { outer radius } r_{\text {out }} \\
(\mathrm{pc})\end{array}$ & $\begin{array}{c}\text { density } n_{c} \\
\left(\mathrm{~cm}^{-3}\right)\end{array}$ \\
\hline Cygnus Rift & $1.26 \times 10^{6}$ & 112 & 662 & 738 & 29 \\
Cygnus OB7 & $1.10 \times 10^{6}$ & 46 & 739 & 861 & 29
\end{tabular}

very low accretion rates, the accretion flow can be stopped at several Thomson depths and the resulting spectrum can be thermalized at a temperature approximately equal to the star effective temperature

$$
\begin{aligned}
T_{e f f}= & \left(\frac{L}{4 \pi r_{*}^{2} f_{s} \sigma}\right)^{1 / 4} \simeq 90\left(\frac{f_{s}}{10^{-3}}\right)^{-1 / 4} \\
& \left(\frac{r_{*}}{10^{6} \mathrm{~cm}}\right)^{-1 / 2}\left(\frac{M_{*}}{M_{\odot}}\right)^{1 / 2} \\
& \left(\frac{n}{1 \mathrm{~cm}^{-3}}\right)^{1 / 4}\left(\frac{v}{40 \mathrm{~km} \mathrm{~s}^{-1}}\right)^{-3 / 4} \mathrm{eV}
\end{aligned}
$$

where $f_{s}$ is the fraction of the surface area which undergoes accretion (see below). Then, ONSs accreting directly from the ISM emit typically in the ultraviolet and soft $\mathrm{X}_{-}$ ray bands. The low bolometric luminosity $\left(L \sim 10^{30}-10^{31}\right.$ erg s$\left.{ }^{-1}\right)$ and the softness of the spectrum $\left(T_{\text {eff }} \sim 90 \mathrm{eV}\right)$ explain the difficulty in observing an isolated ONS. Previous investigations were restricted to the calculation of the emitted spectrum at high luminosities. A detailed numerical analysis of the spectral properties of unmagnetized neutron stars accreting well below the Eddington limit has been recently presented by Zampieri et al. (1995). The emergent spectrum turns out to be significantly hardened with respect to a black body at the star effective temperature, with a broad maximum shifted toward higher frequencies by a factor $\sim 3$ at $L \sim 10^{30} \mathrm{erg} \mathrm{s}^{-1}$. This fact is due entirely to the frequency dependence of the free-free opacity, for which higher energy photons decouple at larger depths and temperatures in the neutron star atmosphere.

In the following, we will consider two possibilities for the emitted flux: first, black body emission at the neutron star effective temperature (see equation 2) which has been frequently used in the previous investigations and can be regarded as a useful approximation; second, the spectra computed by Zampieri et al. (1995). In addition, we assume that a relic magnetic field is present and that the accretion flow is channeled into the polar caps. If we neglect all the radiative effects produced by the magnetic field (on this regard see e.g. Miller 1992; Shibanov et al. 1992; Nelson et al. 1995), the main consequence is to limit the size of the emitting region by a factor $f_{s}=A_{c} / 2 \pi r_{*}^{2}$, where $A_{c}=\pi r_{*}^{3} / r_{A}$ is the area of the polar cap and $r_{A}$ is the Alfvén radius. For $B \simeq 10^{9} \mathrm{G}$, as we consider here, $f_{s} \simeq 10^{-3}$. This fact will produce a hardening of the spectrum with respect to the unmagnetized case with the same luminosity, since the flux emitted per unit surface is $F_{\text {mag }}=L / 2 A_{c}>F_{\text {unmag }}=L / 4 \pi r_{*}^{2}$.

\subsection{Expected number of ONSs}

The technique which has been used to calculate the expected number of ONSs in the molecular clouds is the generalization of a similar procedure introduced by Zane et al. (1995b) and is described in detail in the Appendix. With this technique it is possible to calculate also the expected number of foreground and background ONSs accreting from the average ISM, which are seen in the direction of the clouds but are not embedded within them. This aspect deserves particular notice since, as shall see, they are expected to be quite numerous at low sensitivity limits and could be distinguished from the ONSs embedded in the molecular clouds only by means of their observed spectral properties.

The characteristic parameters of the two clouds are quoted in Table 1. Results of the expected number of sources observable in Cygnus Rift, Cygnus OB7 and also in other spatial regions in the cloud directions are presented in Tables 2 and 3 (fifth column) for different values of the sensitivity limit $S\left(N_{O N S}^{t o t}=10^{9}, B=10^{9} \mathrm{G}\right)$. These numbers are calculated assuming the spectrum of Zampieri et al. (1995) and a black body spectrum (values in brackets). The fraction of clouds which has been covered by the ROSAT pointings at a given sensitivity depends mainly on the exposure time. In the second column of Tables 2 and 3 we quote the fractional coverage of the cloud areas $f$ as a function of threshold. As can be seen, the covering increases from $\sim 1 \%$ at $10^{-3} \mathrm{c} \mathrm{s}^{-1}$ to $\sim 5 \%$ at $10^{-2} \mathrm{c} \mathrm{s}^{-1}$. To compare the theoretical estimates with the actual number of unidentified X-ray sources detected in the ROSAT pointings of our sample, for each interval of distance we have computed the expected number of detectable sources according to the following formula

$$
\begin{aligned}
N\left(>S_{i}\right)_{\left[d_{j}, d_{j+1}\right]}= & \left(N_{\text {ONS }}^{\text {theor }}\right)_{\left[d_{j}, d_{j+1}\right]}^{i} f_{i} \\
& +\left(N_{\text {ONS }}^{\text {theor }}\right)_{\left[d_{j}, d_{j+1}\right]}^{i+1}\left(f_{i+1}-f_{i}\right)+ \\
& +\ldots+ \\
& +\left(N_{\text {ONS }}^{\text {theor }}\right)_{\left[d_{j}, d_{j+1}\right]}^{i_{\max }}\left(f_{i_{\text {max }}}-f_{i_{\text {max }}-1}\right)(, 3)
\end{aligned}
$$

where $\left(N_{O N S}^{\text {theor }}\right)_{\left[d_{j}, d_{j+1}\right]}^{i}$ is the expected number of ONSs above threshold $S_{i}$ detectable in the direction of the whole cloud areas and $f_{i}$ is the fractional coverage of the pointings at the same threshold (see Tables 2 and 3 ). Here $S_{1}=10^{-3} \mathrm{c} \mathrm{s}^{-1}, S_{2}=2 \times 10^{-3} \mathrm{c} \mathrm{s}^{-1}, S_{3}=4 \times 10^{-3}$ 
Table 2. Expected number of ONSs detectable in the direction of Cygnus Rift as a function of threshold $\left(N_{O N S}^{t o t}=10^{9}, B=10^{9}\right.$ $\mathrm{G}$ ). Values in the second column (hardness ratio $H R$ ) and in the last two columns refer to the Zampieri's spectrum and to a black body (values in brackets). $d_{j} \div d_{j+1}$ is the interval of distance considered, $\left(N_{O N S}^{\text {theor }}\right)_{\left[d_{j}, d_{j+1}\right]}$ the expected number of ONSs above threshold $S$ detectable in the direction of the whole cloud area (see the Appendix) and $N(>S)_{\left[d_{j}, d_{j+1}\right]}$ the expected number of sources in the fraction of the cloud $f$ covered by ROSAT pointings (see equation (3)).

\begin{tabular}{|c|c|c|c|c|c|}
\hline $\begin{array}{l}\text { threshold } S \\
\left(\mathrm{c} \mathrm{s}^{-1}\right)\end{array}$ & $\begin{array}{c}\text { coverage } f \\
(\%)\end{array}$ & hardness ratio $H R$ & $\begin{array}{l}d_{j} \div d_{j+1} \\
\quad(\mathrm{pc})\end{array}$ & $\left(N_{O N S}^{\text {theor }}\right)_{\left[d_{j}, d_{j+1}\right]}$ & $N(>S)_{\left[d_{j}, d_{j+1}\right]}$ \\
\hline \multirow[t]{6}{*}{0.001} & 0.63 & & & & \\
\hline & & $-0.86 \div 0.85(-0.98 \div 0.69)$ & $30 \div 100$ & $3(3)$ & $0(0)$ \\
\hline & & $0.06 \div 0.94(-0.85 \div 0.90)$ & $100 \div 300$ & $64(43)$ & $1(1)$ \\
\hline & & $0.82 \div 0.97(0.45 \div 0.95)$ & $300 \div 662$ & $270(113)$ & $7(2)$ \\
\hline & & $0.94 \div 0.99(0.87 \div 0.99)$ & $662 \div 738[\mathrm{Rift}]$ & $297(215)$ & $11(6)$ \\
\hline & & $0.99(0.98 \div 0.99)$ & $738 \div 2500(738 \div 2080)$ & $360(127)$ & $5(2)$ \\
\hline \multirow[t]{6}{*}{0.002} & 2.52 & & & & \\
\hline & & $-0.81 \div 0.85(-0.98 \div 0.69)$ & $30 \div 100$ & $3(3)$ & $0(0)$ \\
\hline & & $0.07 \div 0.94(-0.83 \div 0.90)$ & $100 \div 300$ & $52(33)$ & $1(1)$ \\
\hline & & $0.84 \div 0.97(0.53 \div 0.95)$ & $300 \div 662$ & $167(73)$ & $6(2)$ \\
\hline & & $0.95 \div 0.99(0.89 \div 0.99)$ & $662 \div 738[$ Rift $]$ & $250(170)$ & $10(6)$ \\
\hline & & $0.99(0.98 \div 0.99)$ & $738 \div 2000(738 \div 1630)$ & $152(52)$ & $4(1)$ \\
\hline \multirow[t]{6}{*}{0.004} & 3.15 & & & & \\
\hline & & $-0.74 \div 0.85(-0.97 \div 0.69)$ & $30 \div 100$ & $3(3)$ & $0(0)$ \\
\hline & & $0.17 \div 0.94(-0.79 \div 0.90)$ & $100 \div 300$ & $39(24)$ & $1(1)$ \\
\hline & & $0.86 \div 0.97(0.62 \div 0.95)$ & $300 \div 662$ & $96(46)$ & $4(1)$ \\
\hline & & $0.95 \div 0.99(0.90 \div 0.99)$ & $662 \div 738$ [Rift] & $191(124)$ & $9(5)$ \\
\hline & & $0.99(0.98 \div 0.99)$ & $738 \div 1500(738 \div 1260)$ & $47(17)$ & $1(1)$ \\
\hline \multirow[t]{6}{*}{0.008} & 4.41 & & & & \\
\hline & & $-0.64 \div 0.85(-0.96 \div 0.69)$ & $30 \div 100$ & $3(3)$ & $0(0)$ \\
\hline & & $0.33 \div 0.94(-0.68 \div 0.90)$ & $100 \div 300$ & $26(16)$ & $1(1)$ \\
\hline & & $0.88 \div 0.97(0.69 \div 0.95)$ & $300 \div 662$ & $51(27)$ & $2(1)$ \\
\hline & & $0.96 \div 0.99(0.92 \div 0.99)$ & $662 \div 738$ [Rift] & $129(85)$ & $7(4)$ \\
\hline & & $0.99(0.98 \div 0.99)$ & $738 \div 1100(738 \div 960)$ & $12(4)$ & $1(0)$ \\
\hline \multirow[t]{6}{*}{0.01} & 5.04 & & & & \\
\hline & & $-0.60 \div 0.85(-0.96 \div 0.69)$ & $30 \div 100$ & $2(2)$ & $0(0)$ \\
\hline & & $0.35 \div 0.94(-0.64 \div 0.90)$ & $100 \div 300$ & $23(14)$ & $1(1)$ \\
\hline & & $0.89 \div 0.97(0.71 \div 0.95)$ & $300 \div 662$ & $42(23)$ & $2(1)$ \\
\hline & & $0.96 \div 0.99(0.92 \div 0.99)$ & $662 \div 738$ [Rift] & $110(74)$ & $6(4)$ \\
\hline & & $0.99(0.98 \div 0.99)$ & $738 \div 1030(738 \div 880)$ & $8(2)$ & $0(0)$ \\
\hline
\end{tabular}

$\mathrm{c} \mathrm{s}^{-1}, S_{4}=8 \times 10^{-3} \mathrm{c} \mathrm{s}^{-1}, S_{i_{\max }}=S_{5}=10^{-2} \mathrm{c} \mathrm{s}^{-1}$. The first term on the right hand side of equation (3) gives the number of sources observable in the pointings with limiting sensitivity $S_{i}$, while the other terms account for the contributions from pointings at lower sensitivity. Finally, the total expected number of sources $N\left(>S_{i}\right)$ is obtained integrating $N\left(>S_{i}\right)_{\left[d_{j}, d_{j+1}\right]}$ over distance and is shown in Figure 2 (summing the contributions from the two clouds).

In the fraction of the clouds actually observed by ROSAT, we expect the detection of 10-24 sources (the exact value depends on the assumption on the emitted spectrum) at the sensitivity limit of $1-2 \times 10^{-3} \mathrm{c} \mathrm{s}^{-1}$ in the direction of Cygnus Rift and of 5-13 sources in the direction of Cygnus OB7. Among these sources, 6-11 sources are expected to be really embedded in Cygnus Rift and 3-6 in Cygnus OB7. The other objects are foreground or background sources; in particular, we estimate that 3-8 and $2-6$ foreground ONSs should be detectable in the direction of Cygnus Rift and Cygnus OB7, respectively. We note that at high thresholds $10^{-2} \mathrm{c} \mathrm{s}^{-1}$ the detection of a significant number of sources would be expected.

Then, in agreement with previous investigations (Blaes \& Madau 1993; Colpi, Campana \& Treves 1993; Zane et al. 1995a) Cygnus Rift and Cygnus OB7 are expected to be particularly favourable sites for the observability of ONSs with ROSAT PSPC. In addition, we have found that in their direction a significant number of foreground and background ONSs should be observable.

\subsection{Expected emission properties}

In principle we could compare directly the theoretical spectral distribution with the observed one but, as we shall see, the sources selected in our sample have too few photons to extract a spectrum. Then, in order to com- 
Table 3. Expected number of ONSs detectable in the direction of Cygnus OB7 as a function of threshold $\left(N_{O N S}^{t o t}=10^{9}, B=10^{9}\right.$ $\mathrm{G})$. Values in the second column (hardness ratio $H R$ ) and in the last two columns refer to the Zampieri's spectrum and to a black body (values in brackets). $d_{j} \div d_{j+1}$ is the interval of distance considered, $\left(N_{O N S}^{\text {theor }}\right)_{\left[d_{j}, d_{j+1}\right]}$ the expected number of ONSs above threshold $S$ detectable in the direction of the whole cloud area (see the Appendix) and $N(>S)_{\left[d_{j}, d_{j+1}\right]}$ the expected number of sources in the fraction of the cloud $f$ covered by ROSAT pointings (see equation (3)).

\begin{tabular}{|c|c|c|c|c|c|}
\hline \multirow[t]{6}{*}{$\begin{array}{l}\text { threshold } S \\
0.001\end{array}$} & $\begin{array}{l}\text { coverage } f \\
1.55\end{array}$ & hardness ratio $H R$ & $d_{j} \div d_{j+1}$ & $\left(N_{O N S}^{\text {theor }}\right)_{\left[d_{j}, d_{j+1}\right]}$ & $N(>S)_{\left[d_{j}, d_{j+1}\right]}$ \\
\hline & & $-0.86 \div 0.85(-0.98 \div 0.69)$ & $30 \div 100$ & $3(3)$ & $0(0)$ \\
\hline & & $0.06 \div 0.94(-0.85 \div 0.90)$ & $100 \div 300$ & $64(43)$ & $2(1)$ \\
\hline & & $0.82 \div 0.99(0.45 \div 0.99)$ & $300 \div 739$ & $136(56)$ & $4(1)$ \\
\hline & & $0.95 \div 0.99(0.89 \div 0.99)$ & $739 \div 861[\mathrm{OB} 7]$ & $216(140)$ & $6(4)$ \\
\hline & & $0.99(0.99)$ & $861 \div 2100(861 \div 1700)$ & $67(22)$ & $1(0)$ \\
\hline \multirow[t]{6}{*}{0.002} & 2.32 & & & & \\
\hline & & $-0.81 \div 0.85(-0.98 \div 0.69)$ & $30 \div 100$ & $3(3)$ & $0(0)$ \\
\hline & & $0.07 \div 0.94(-0.83 \div 0.90)$ & $100 \div 300$ & $52(33)$ & $2(1)$ \\
\hline & & $0.84 \div 0.99(0.53 \div 0.99)$ & $300 \div 739$ & $82(36)$ & $3(1)$ \\
\hline & & $0.96 \div 0.99(0.90 \div 0.99)$ & $739 \div 861[\mathrm{OB} 7]$ & $167(105)$ & $6(3)$ \\
\hline & & $0.99(0.99)$ & $861 \div 1600(861 \div 1300)$ & $22(7)$ & $1(0)$ \\
\hline \multirow[t]{6}{*}{0.004} & 3.87 & & & & \\
\hline & & $-0.74 \div 0.85(-0.97 \div 0.69)$ & $30 \div 100$ & $3(3)$ & $0(0)$ \\
\hline & & $0.17 \div 0.94(-0.79 \div 0.90)$ & $100 \div 300$ & $39(24)$ & $2(1)$ \\
\hline & & $0.86 \div 0.99(0.62 \div 0.99)$ & $300 \div 739$ & $46(23)$ & $2(1)$ \\
\hline & & $0.96 \div 0.99(0.92 \div 0.99)$ & $739 \div 861[\mathrm{OB} 7]$ & $117(73)$ & $5(3)$ \\
\hline & & $0.99(0.99)$ & $861 \div 1200(861 \div 990)$ & $5(1)$ & $0(0)$ \\
\hline \multirow[t]{6}{*}{0.008} & 3.87 & & & & \\
\hline & & $-0.64 \div 0.85(-0.96 \div 0.69)$ & $30 \div 100$ & $3(3)$ & $0(0)$ \\
\hline & & $0.33 \div 0.94(-0.68 \div 0.90)$ & $100 \div 300$ & $26(16)$ & $1(1)$ \\
\hline & & $0.88 \div 0.99(0.69 \div 0.99)$ & $300 \div 739$ & $25(13)$ & $1(1)$ \\
\hline & & $0.96 \div 0.99(0.93 \div 0.99)$ & $739 \div 861(739 \div 839)[\mathrm{OB} 7]$ & $72(45)$ & $3(2)$ \\
\hline & & 0.99 & $861 \div 914$ & 0 & \\
\hline \multirow[t]{5}{*}{0.01} & 4.64 & & & & \\
\hline & & $-0.60 \div 0.85(-0.96 \div 0.69)$ & $30 \div 100$ & $2(2)$ & $0(0)$ \\
\hline & & $0.35 \div 0.94(-0.64 \div 0.90)$ & $100 \div 300$ & $23(14)$ & $1(1)$ \\
\hline & & $0.89 \div 0.99(0.71 \div 0.99)$ & $300 \div 739$ & $20(11)$ & $1(1)$ \\
\hline & & $0.97 \div 0.99(0.93 \div 0.99)$ & $739 \div 861(739 \div 824)[\mathrm{OB} 7]$ & $65(33)$ & $3(2)$ \\
\hline
\end{tabular}

pare our theoretical expectations with the observations, in each range of distances considered we have simulated PSPC spectra by folding the theoretical model with interstellar absorption, detector effective area and response matrix, and calculated the PSPC hardness ratio, defined by

$H R=\frac{N(41-240)-N(11-40)}{N(41-240)+N(11-40)}$,

where $N(11-40)$ and $N(40-240)$ are the count rates in the PSPC channel ranges 11-40 and 41-240, corresponding roughly to the energy bands $0.1-0.4 \mathrm{keV}$ and $0.4-2.4$ $\mathrm{keV}$. We have repeated the calculation assuming either the spectrum computed by Zampieri et al. (1995) or a black body at the neutron star effective temperature. Results are presented in Tables 2 and 3, where the lowest and highest values of the hardness ratio is reported for each interval of distance (the highest value refers to the model with maximum luminosity). It is interesting to note that, for distances above $\sim 600 \mathrm{pc}$ for the black body and $\sim 300$ pc for the synthetic spectra, the absorption of the interstellar medium and the cloud material causes the hardness ratio to be very close to unity. Then, although ONSs are relatively soft sources, above these distances they should appear significantly hardened.

We have estimated also the apparent visual magnitude of ONSs below $100 \mathrm{pc}$ from the Sun and find that $m_{V} \gtrsim 32$. Then, these sources should lack of optical counterparts in the digitized red plates of the Palomar All Sky Survey (POSS, limiting magnitude $m_{r} \sim 20$ ). According to recent findings by Blaes, Warren \& Madau (1995), for ONSs embedded within the clouds and considering polar cap emission the reprocessing of the UV-soft $\mathrm{X}$-ray radiation by the surrounding gas might increase the emitted flux at optical wavelengths by 1-2 magnitudes. However, even in this case the very low visual magnitude prevents any possibility of optical identification for sources beyond 100 pc. Then, we will use the lack of optical counterparts in the digitized POSS plates as a distinguishing criterion for selecting ONSs candidates among ROSAT sources. 
Inserting in equation (A2) the appropriate effective areas and frequencies (taken from the world wide web site of the Center for Extreme Ultraviolet Astrophysics), we have calculated also the expected count rates in the Lex band (67-178 $\AA$ ) of the Extreme Ultraviolet Explorer Deep Survey (EUVE DS). Below $\sim 250-300 \mathrm{pc}$, the count rate turns out to be smaller than $10^{-5} \mathrm{c} \mathrm{s}^{-1}$ even for the more luminous sources and no detectable UV flux can be observed at Earth.

Finally, comparing the observed hardness ratios with the values quoted in Tables 2 and 3 could be used to discriminate ONSs among other optically unidentified X-ray ROSAT sources.

\section{Data analysis}

\subsection{The sample}

We selected from the ROSAT Public Data archive PSPC pointings in the direction of the Cyg OB7 and Cyg Rift molecular clouds. A description of the satellite and the detector can be found in Trümper (1983) and Pfeffermann et al. (1986). The 28 analyzed areas are shown in Figure 1 , overlaid onto the CO contour maps from Dame \& Thaddeus (1985). The relevant data on the pointings are reported in Table 4. A few pointings lie just outside the contour of the clouds in Figure 1, but have been nevertheless included in the sample. The total exposure time is 126603 seconds. Only the central region of the PSPC (radius $20^{\prime}$ ) has been considered for the analysis, to avoid problems due to the poor knowledge of the point spread functions outside that region. The areas of the two clouds covered by ROSAT pointings are 2.1 square degrees (Cyg OB7) and 5.6 square degrees (Cyg Rift), which correspond to $4.6 \%$ and $5.0 \%$ of the total areas of the clouds, respectively (adopting the values quoted in Dame \& Thaddeus 1985). Because of the nature of the pointings, the fractional coverage of the cloud areas is limited and varies with threshold (see Tables 2 and 3). Although at the lowest sensitivity limits $\left(1-21^{-3} \mathrm{c} \mathrm{s}^{-1}\right)$ only $1-2 \%$ has been covered, the theoretical expectations (see again Tables 2 and 3) indicate that this could be sufficient for detecting ONS candidates. Moreover, from the systematic analysis of these data a search methodology can be outlined.

\subsection{The analysis procedure}

Each pointing has been extracted from the ROSAT Public Archive at MPE Garching and analyzed separately by running a set of semi-automatic programs. The standard detection technique in the EXSAS package (Zimmermann et al. 1994) has been applied. First a background map is produced by removing all possible sources (identified by means of a sliding window technique) and running a two dimensional spline fit to the data. Then a Maximum Likelihood (ML) algorithm is applied (see Cruddace, Hasinger \& Schmitt 1988) to detect significant deviations from the
Table 4. Observation log. The first two columns are the identifier of the pointing in this paper and the ROSAT observation number. Galactic coordinates are in decimal degrees. $\mathrm{N}_{X}$ is the number of detected $\mathrm{X}$-ray sources and $\mathrm{N}_{G S C}$ is the number of GSC stars used for the boresight correction (noB indicates one star or less, see text).

\begin{tabular}{|c|c|c|c|c|c|c|}
\hline Label & ROR \# & Exp. (s) & $l_{I I}$ & $\mathrm{~b}_{I I}$ & $\mathrm{~N}_{X}$ & $\mathrm{~N}_{G S C}$ \\
\hline \multicolumn{7}{|c|}{ Cyg OB7 } \\
\hline A & 200427 & 382.0 & 91.4 & 1.1 & 1 & noB \\
\hline K & 500143 & 8260.0 & 93.3 & 6.9 & 9 & 4 \\
\hline $\mathrm{L}$ & 500254 & 4297.0 & 93.7 & -0.3 & 1 & noB \\
\hline S & 300033 & 4331.0 & 89.8 & -0.1 & 5 & noB \\
\hline $\mathrm{T}$ & 300033_1 & 2854.0 & 89.8 & -0.1 & 6 & 3 \\
\hline W & 500195 & 9137.0 & 94.0 & 1.0 & 7 & 5 \\
\hline $\mathrm{b}$ & 900194 & 4166.0 & 89.4 & -0.7 & 3 & 2 \\
\hline \multicolumn{7}{|c|}{ Cyg Rift } \\
\hline B & 200057 & 1846.0 & 76.6 & 1.4 & 4 & 3 \\
\hline $\mathrm{C}$ & 200058 & 1931.0 & 76.6 & 1.4 & 2 & 2 \\
\hline D & 200062 & 14030.0 & 76.6 & 1.4 & 17 & 14 \\
\hline $\mathrm{E}$ & 200063 & 2073.0 & 76.6 & 1.4 & 3 & 3 \\
\hline $\mathrm{F}$ & 200109 & 3587.0 & 80.2 & 0.8 & 11 & 9 \\
\hline G & 200114 & 3508.0 & 85.7 & 1.5 & 1 & noB \\
\hline $\mathrm{H}$ & 200564 & 8950.0 & 74.3 & 1.1 & 5 & 3 \\
\hline I & 500084 & 2470.0 & 69.7 & 1.0 & 2 & 2 \\
\hline $\mathrm{J}$ & 500085 & 2879.0 & 83.0 & -0.3 & 2 & 2 \\
\hline M & 900157 & 4054.0 & 78.2 & 2.0 & 2 & 1 \\
\hline $\mathrm{N}$ & 900158 & 4123.0 & 76.0 & 0.4 & 1 & noB \\
\hline $\mathrm{O}$ & 200154 & 1261.0 & 75.8 & 1.3 & 0 & - \\
\hline $\mathrm{P}$ & 200412 & 1265.0 & 78.2 & 0.1 & 1 & noB \\
\hline Q & 200412_1 & 2228.0 & 78.2 & 0.1 & 2 & noB \\
\hline $\mathrm{R}$ & 200702 & 4409.0 & 85.7 & -0.3 & 7 & 5 \\
\hline U & 400157 & 2996.0 & 77.0 & 3.2 & 3 & 2 \\
\hline V & 500124 & 5979.0 & 70.7 & 1.2 & 1 & noB \\
\hline $\mathrm{X}$ & 500203 & 3641.0 & 84.2 & -0.8 & 1 & noB \\
\hline Y & 500206 & 10323.0 & 78.4 & 2.8 & 13 & 6 \\
\hline Z & 500207 & 9319.0 & 77.9 & 3.5 & 8 & 5 \\
\hline$\underline{a}$ & 500208 & 2304.0 & 77.5 & 4.0 & 2 & noB \\
\hline$\overline{\text { Total }}$ & & 126603 & & & 120 & \\
\hline
\end{tabular}

estimated background distribution. The threshold for detection has been set to the conventional value of $\mathrm{ML}=10$, which corresponds to a chance detection probability of $4.5 \times 10^{-5}$ for a single trial. This technique is repeated for the data in three different PHA channel ranges: total ( $\mathrm{T}$ : channels 11-240, 0.1-2.4 keV), soft (S: channels 11-40, 0.1-0.4 keV) and hard (H: channels 41-240, 0.4-2.4 keV). In case of a detection in more than one band, only the band with the highest value of ML has been considered.

It is known that the ML technique becomes problematic in presence of large regions of extended emission, as it is likely to find in the pointing directions considered here, lying on the galactic plane. Therefore, all the detected sources have been visually inspected in the X-ray images and obvious spurious detections have been discarded. 
Fig. 1. Map of the analyzed ROSAT PSPC pointings. Each circle is $20^{\prime}$ radius. The black circles correspond to pointings containing ONS candidates (see text). The contours of the clouds are from Dame \& Thaddeus (1985).

Fig. 2. The $\log N-\log S$ distribution for all the sources detected by ROSAT (continuous line) and all the optically unidentified sources detected by ROSAT (dashed line). Also shown is the expected number of ONSs, $N(>S)$, in the fraction of the clouds covered by ROSAT pointings (dash-dotted line; Zampieri's spectrum).

In the cases where two or more pointings were coaligned, they were analyzed separately: the sources have been cross-correlated and in case of coincidences only the source with the highest detection ML has been considered. Eleven sources have been eliminated in this way, bringing the total number of sources to 109 . The $\log N-\log S$ dis- tribution for the sources detected by ROSAT is shown in Figure 2. There are a few strong sources, but most of them are below $0.01 \mathrm{c} \mathrm{s}^{-1}$. Note that the targets of the pointings have not been removed.

\subsection{Optical identification}

The positions of the detected X-ray sources had to be corrected for an offset due to a residual systematic uncertainty in the position determination (the so-called boresight correction). In order to do this, preliminary identifications have been performed using the HST Guide Star Catalog (GSC; Lasker et al. 1990). The X-ray sources and GSC stars have been cross-correlated and the best shifts to the $\mathrm{X}$-ray positions have been determined by means of a Maximum Likelihood technique. The shift so obtained has been considered only if at least two sources were identified in this way (with the exception of pointing $\mathrm{S}$, where one of the two sources identified with GSC entries had multiple bright optical counterparts). For the remaining pointings, a $6^{\prime \prime}(1 \sigma)$ error has been quadratically added to the error radii of the detected sources (Kürster \& Hasinger 1992). In Table 4 a summary of the X-ray detections and boresight corrections is reported. An X-ray source is considered to be identified if a GSC star falls into its $90 \%$ positional error circle. In case of more than one optical identification, the brightest counterpart has been considered.

$67 \mathrm{X}$-ray sources were identified with entries in the GSC, which has a loosely defined magnitude limit of $m_{V} \sim 14$. For the remaining 42 sources, we used the Digitized Sky Survey (DSS; Postman et al. 1995), which consists of the POSS red plates. The magnitudes of the brightest stars in the error boxes have been estimated by using 
the empirical magnitude-diameter relation of King \& Raff (1977) appropriate for red POSS plates. The values so obtained have a large uncertainty (up to one magnitude), but it is sufficient for our purpose. Only in three cases the possible optical counterpart was not bright enough to allow an estimate of the magnitude, the image being not saturated. In Figure 3 we show the distribution of magnitudes of the optical counterparts for the two samples, GSC and DSS. As one can observe there is a considerable number of bright stars, since there are bright OB associations in the direction of most of the pointings; indeed a large number of the counterparts for which we were able to obtain a spectral type were of types $\mathrm{O}$ and $\mathrm{B}$. The presence of strong optical candidates for many $\mathrm{X}$-ray sources ensures the robustness of the boresight process.

After the optical screening, 7 sources were left for which no optical counterpart could be seen in the POSS red plates, to a limiting magnitude $m_{r} \sim 20$. Two of these were coincident with the original targets of the pointings and were therefore excluded. The brightest of the remaining sources was subsequently identified with $\mathrm{Cyg} \mathrm{X}-3$, so 4 final candidates remained.

\subsection{ONS candidates}

For the four sources with no optical counterpart, PSPC count rates in the different bands have been extracted manually from the data, with an accurate estimate of the background, in some case contaminated by extended emission. The relevant information about the candidates (two in each cloud) can be found in Table 5. As it can be seen from Table 4, all four sources come from pointings with long exposure times and with a large number of GSC identifications, which ensures an accurate boresight correction. Source OB7-2 lies just outside the outer rim of the cloud (see Figure 1), but we decided not to discard it from the sample.

Source Rift-2 might have counterparts at different wavelengths. The POSS plate shows a very faint ringlike structure $\left(\sim 20^{\prime \prime}\right.$ diameter $)$ in the $\mathrm{X}$-ray error box, reminiscent of a supernova remnant or a planetary nebula. The position of the source is compatible with that of the IRAS source $20179+4102$, which has a $100 \mu \mathrm{m}$ flux of $75 \mathrm{Jy}$ (IRAS Catalogs and Atlases 1985). Putting the distance at $0.7 \mathrm{kpc}$, this translates roughly to a luminosity of $\sim 10^{35} \mathrm{erg} \mathrm{s}^{-1}$. At the same distance the radius of the optical ring would be $\sim 0.1 \mathrm{pc}$. If the ring and the IRAS sources were the same object, it would be too small and faint to be a supernova remnant, while the planetary nebula hypothesis could not be ruled out. In absence of a clear identification we do not exclude the source from our candidates list.
Fig. 3. Distribution of optical magnitudes for the possible counterparts of the X-ray sources. GSC magnitudes are in the $V$ band, POSS magnitudes in the red (see text). The lack of POSS stars at magnitude 19 is a probable artifact of the procedure for magnitude estimate.

\section{Discussion}

As can be seen from Tables 2, 3 and 5, our predicted number of sources is larger than the number of unidentified objects detected by ROSAT. In particular, Figure 2 shows the expected number of ONSs and the observed number of unidentified objects in our sample as a function of threshold. By comparing the two curves, it is clearly visible that predictions are too "optimistic", although it should be stressed that, by purpose, theoretical calculations have usually been performed under the most favourable assumptions. The fact that the observed number of ONS candidates is smaller by a factor $3-10$ indicates that the range of parameters of our model needs to be more constrained. As already discussed, there are several sources of uncertainties which may influence theoretical predictions, such as the velocity distribution and density of ONSs, the strength of the relic magnetic field (related to the possible inhibition of accretion), the neutron star birth rate, the total number of ONSs, the indetermination in the exact value of the gravitational capture radius $r_{g c}$ and the validity of the fluid approximation for the average ISM. Scattering by molecular clouds and spiral arms (dynamical heating; Wielen 1977) may boost a fraction of low velocity stars to higher speeds, reducing the number counts up to an order of magnitude (Madau \& Blaes 1994). Also, evidence has been found (Lyne \& Lorimer 1994) for a neutron star mean birth velocity much higher than the one obtained by Narayan \& Ostriker (1990). Recently, Blaes, Warren \& Madau (1995) have shown that preheating of the ambient gas to temperatures higher than $10^{4} \mathrm{~K}$ may 
T. Belloni et al.: Search for Old Neutron Stars in molecular clouds: Cygnus Rift and Cygnus OB7

Table 5. ONS candidates. The columns are: source name, pointing identifier, celestial coordinates, 90\% error radius, Maximum Likelihood of existence, PSPC total count rate (channels 11-240) and hardness ratio (defined as $H R=(\mathrm{H}-\mathrm{S}) /(\mathrm{H}+\mathrm{S})$, where $\mathrm{S}$ and $\mathrm{H}$ are the counts in the channel intervals $11-40$ and $41-240$ respectively.

\begin{tabular}{cccccccc} 
Name & $\mathrm{P}$. & $\alpha(2000)$ & $\delta(2000)$ & $\Delta r\left(^{\prime \prime}\right)$ & $\mathrm{ML}$ & $\mathrm{c} \mathrm{ksec}^{-1}$ & $H R$ \\
\hline \hline Rift-1 & $\mathrm{D}$ & $20^{h} 19^{m} 27.96^{s}$ & $+38^{\circ} 38^{\prime} 27.6^{\prime \prime}$ & 10.5 & 24.7 & $2.7 \pm 0.6$ & $0.29 \pm 0.23$ \\
Rift-2 & $\mathrm{Y}$ & $20^{h} 19^{m} 47.05^{s}$ & $+41^{\circ} 12^{\prime} 01.3^{\prime \prime}$ & 19.2 & 10.9 & $3.2 \pm 0.9$ & $>0.28$ \\
OB7-1 & $\mathrm{K}$ & $20^{h} 53^{m} 07.35^{s}$ & $+55^{\circ} 13^{\prime} 29.0^{\prime \prime}$ & 13.4 & 17.7 & $1.5 \pm 0.5$ & $>0.44$ \\
OB7-2 & $\mathrm{W}$ & $21^{h} 25^{m} 31.12^{s}$ & $+51^{\circ} 48^{\prime} 31.5^{\prime \prime}$ & 9.2 & 35.2 & $2.4 \pm 0.6$ & $>0.84$
\end{tabular}

significantly reduce the accretion rate, hence decreasing the number of detectable objects.

Our ONSs searching strategy was aimed to select $\mathrm{X}^{-}$ ray objects in molecular clouds which, within their error boxes, do not have any optical counterpart in the POSS plates. Additional information is clearly needed to assess the true nature of these objects. The detection of an $\mathrm{X}_{-}$ ray pulsation in the range of $1-1000 \mathrm{~s}$ will clearly indicate that we are observing an ONS; however high magnetic fields are needed which could prevent accretion and could harden substantially the emitted spectrum (see e.g. Nelson et al. 1995), moving the peak emissivity outside the ROSAT energy range. In absence of X-ray pulsations, a spectral criterion could be used to individuate ONSs: in the case of low magnetized or unmagnetized ONSs we expect an X-ray spectrum which show a significant hardening with respect to a black body at the neutron star effective temperature, as discussed in Zampieri et al. (1995). An additional feature could be a high column density, testifying that the $\mathrm{X}$-ray object lies within (or beyond) the molecular cloud. As can be seen from Tables 2 and 3, the expected hardness ratios for ONSs embedded in the clouds or beyond them are near to unity. In this respect, the observed $H R$ of three of our candidates are consistent with the hypothesis that the sources are located within the clouds, whereas one source (Rift-1) cannot certainly lie within the cloud because of its measured value of the hardness ratio $H R=0.29 \pm 0.23$ (see Table 5). However, it should be noted that its spectral properties are consistent with those of a foreground ONS located at $\gtrsim 100-300 \mathrm{pc}$ (see also Table $2 \mathrm{a}, S=2 \times 10^{-3} \mathrm{c} \mathrm{s}^{-1}$ ). We checked that, at this distances, Rift-1 should not be detectable even at the lowest sensitivity limits $\left(\gtrsim 10^{-3} \mathrm{c} \mathrm{s}^{-1}\right)$ of EUVE DS.

From the lack of optical counterparts in the digitized POSS plates, we can estimate a lower limit for the observed X-ray to optical flux ratio $\left(F_{X} / F_{V}\right)$ for our ONS candidates. The limiting magnitude in the regions of the four candidates is $m_{r} \sim 20$; using the conversion to magnitude $m_{V}$ by King and Raff (1977) we can adopt this limit as a conservative limit on $m_{V}$, since only blue objects would break this assumption and the bluest unabsorbed stars have $\mathrm{B}-\mathrm{V}=-0.3$. For the conversion between ROSAT countrate and flux we adopted the relation by Fleming et al. (1995), appropriate for sources of stellar nature. The resulting values for $\log F_{X} / F_{V}$ are between -0.4 and +0.1 .

Stocke et al. (1995) use the value of $F_{X} / F_{V}$ as a chief criterion for determining the nature of the $\mathrm{X}$-ray emitter. In addition to compact objects, high $F_{X} / F_{V}$ are associated also with BL Lacs, cluster of galaxies, young supernova remnant and heavily extincted pre-main sequence stars. While an extragalactic origin seems unlikely for sources on the galactic plane in the direction of molecular clouds, the other possibilities still cannot be excluded.

Follow-up deep observations will be necessary to further investigate the nature of these ONS candidates. In particular, deep X-ray pointings in the direction of these four sources would be very useful to measure with more precision the X-ray spectral properties. Also, UV observations in the LeX band of EUVE at the lowest sensitivity limits would be desirable to verify the absence of ultraviolet emission. Finally, deep optical observations are certainly needed to search for the presence of possible fainter counterparts within their error circle and to put a better constraint on the $F_{X} / F_{V}$ ratio.

\section{A. Appendix: expected number of ONSs}

In the calculation of the expected number of ONSs we have used the analytic approximation to the ONS distribution computed by Zane et al. (1995a), which is based on the evolution of the $\mathrm{F}$ population (characterized by the lowest value of the mean velocity) of model $b$ of Narayan \& Ostriker (1990) and on the assumption of a uniform spatial distribution of neutron stars:

$$
\begin{aligned}
\frac{d N_{O N S}}{d V d v} & =n_{O N S} \frac{d G(v)}{d v} \\
& =n_{O N S} \frac{d}{d v}\left[\frac{\left.v / 69 \mathrm{~km} \mathrm{~s}^{-1}\right)^{3.3}}{1+\left(v / 69 \mathrm{~km} \mathrm{~s}^{-1}\right)^{3.3}}\right]
\end{aligned}
$$

where $d V$ is the spatial volume integration element, $n_{O N S}=3 \times 10^{-4}\left(N_{O N S}^{t o t} / 10^{9}\right) \mathrm{pc}^{-3}$ is the number density of ONSs averaged in the local region around the Sun $(r \leq 2 \mathrm{kpc},|z| \leq 200 \mathrm{pc}), N_{O N S}^{t o t}$ is the present total number of ONSs in the Galaxy and $G(v)$ is an analytic fit to the cumulative velocity distribution derived by Zane et al. (1995a). 
The count rate (measured at Earth) in a certain spectral interval $\left(\nu_{1}, \nu_{2}\right)$ of a star which emits a monochromatic flux of radiation $F_{\nu}$ is

$\tilde{N}\left(\nu_{1}, \nu_{2}\right)=\int_{\nu_{1}}^{\nu_{2}} f_{s} \frac{F_{\nu}}{h \nu}\left(\frac{r_{*}}{d}\right)^{2} e^{-\sigma_{\nu} N_{H}} A_{\nu} d \nu$

where $d$ is the distance of the source, $\sigma_{\nu}$ is the absorption cross-section of the ISM (Morrison \& McCammon 1983) and $N_{H}$ the hydrogen column density. $A_{\nu}$ is the effective area and $\Delta \nu=\nu_{2}-\nu_{1}$ the bandpass of the detector $\left(\nu_{1}=\right.$ $0.1 \mathrm{keV}$ and $\nu_{2}=2.4 \mathrm{keV}$ for ROSAT PSPC).

Clearly, at a certain distance $d_{j}$ an ONS will be detectable if its count rate $\widetilde{N}$ is above the sensitivity limit $S$ of the detector. Then, at $d_{j}$ there exists a limiting value of the star luminosity, $\left(L_{m i n}\right)_{d_{j}}$ which depends on the emission properties and the absorption of the ISM, below which an accreting neutron star does not give rise to a count rate above the threshold of the detector and hence is not observable. From equation (1) this translates directly into an upper limit for the star velocity, $\left(v_{\max }\right)_{d_{j}}$.

Alternatively, a star with a given luminosity $L$, or velocity $v$, will be observable up to a maximum distance $d_{\max }(v)$ at which $\widetilde{N}$ goes below $S$. Then, the total number of ONSs which can be observed in a certain interval of distance $\left[d_{j}, d_{j+1}\right]$ and within a solid angle $\Sigma$ can be calculated from equation (A1) summing up all the neutron stars that are contained within the volume $V(v)=\Sigma\left[d_{\max }^{3}(v)-d_{j}^{3}\right] / 3$ and integrating over $v$ :

$$
\begin{aligned}
\left(N_{O N S}^{\text {theor }}\right)_{\left[d_{j}, d_{j+1}\right]}= & n_{O N S} \int_{v_{j+1}}^{v_{j}} V(v) \frac{d G}{d v} d v+ \\
& +n_{O N S} V\left(v_{j+1}\right) G\left(v_{j+1}\right)
\end{aligned}
$$

In equation (A3) the integral goes from the maximum velocity of detectability at distance $d_{j+1}, v_{j+1}=\left(v_{\max }\right)_{d_{j+1}}$, to the maximum velocity at $d_{j}, v_{j}=\left(v_{\max }\right)_{d_{j}}$, whereas the second term on the right hand side accounts for all the stars with luminosity above threshold throughout all of the spatial volume considered. The integral has been evaluated numerically using a Lobatto quadrature and $d_{\max }(v)$ has been interpolated at the appropriate value of $v$ from a table of entries previously calculated (an extensive use has been done of a local cubic interpolation procedure). We note that for each threshold there exists an absolute upper limit to the distance of detectability which corresponds to the star with the minimum accretion velocity $v=v_{s}$ and maximum emitted luminosity. We note also that in each interval of distance $\left[d_{j}, d_{j+1}\right]$ the ISM density must be constant in order to ensure the regularity of the function $d_{\max }(v)$ and hence the correct computation of the integral.

We have estimated the spatial boundaries $r_{i n}=r_{c}-$ $\Delta r_{c}$ and $r_{\text {out }}=r_{c}+\Delta r_{c}$ of Cygnus Rift and Cygnus OB7 ( $r_{c}$ is the position of the cloud center) by inverting for the cloud width $\Delta r_{c}$ the expression $V_{c}=\Sigma_{c}\left(r_{\text {out }}^{3}-r_{i n}^{3}\right) / 3$, where $V_{c}$ is the cloud volume and $\Sigma_{c}$ its apparent angular surface (taken from Dame et al. 1987; see also Table 1). Using these boundaries, the expected number of ONSs in each cloud has been evaluated from equation (A3). Moreover, with the same technique, it is possible to calculate also the expected number of foreground and background ONSs accreting from the average ISM, which are seen in the direction of the clouds but are not embedded within them.

Acknowledgements. We would like to thank R. Waters and A. Treves for useful discussions. TB is supported by NWO under grant PGS 78-277; SC gratefully acknowledges the receipt of an Italian Space Agency fellowship. This research was supported in part by NSF grant AST 93-15133 and NASA grant 5-2925 at the University of Illinois.

\section{References}

Alme M.L. \& Wilson J.R., 1973, ApJ, 186, 1015

Blaes O. \& Madau P., 1993, ApJ, 403, 690

Blaes O. \& Rajagopal M., 1991, ApJ, 381, 210

Blaes O., Warren O. \& Madau P., 1995, ApJ, 454, 370

Colpi M., Campana S. \& Treves A., 1993, A\&A, 278, 161

Cruddace R.G., Hasinger G.R. \& Schmitt J.H., 1988, in "Astronomy from large databases", eds. F. Murtagh \& A. Heck, 177

Dame T.M. \& Thaddeus P., 1985, ApJ, 297, 751

Dame T.M. et al., 1987, ApJ, 322, 706

Diamond C.J., Jewell S.J. \& Ponman T.J., 1995, MNRAS, 274, 589

Dickey J.M. \& Lockman F.J., 1990, ARA\&A, 28, 215

Fleming T.A., Molendi S., Maccacaro T. \& Wolter A., 1995, ApJSS, 99, 701

Frisch P.C. \& York D.G., 1983, ApJ, 271, L59

Hartmann D., Epstein R.I. \& Woosley S.E., 1990, ApJ, 348, 625

Helfand D.J., Chanan G.A. \& Novick R., 1980, Nature, 283, 337

Illarionov A.F. \& Sunyaev R.A., 1975, A\&A, 39, 185

IRAS Catalogs and Atlases, 1985, eds. C.A. Beichman et al., NASA RP-1190

King I.R. \& Raff M.I., 1977, PASP, 89, 120

Koester D., 1976, A\&A, 52, 415

Kürster M. \& Hasinger G., 1992, MPE Memo TN-ROS-MEZA00/028

Lasker B.M., Sturch C.R., McLean B.J., Russell J.L., Jenkner H. \& Shara M.M., 1990, AJ, 99, 2019

Lipunov V.M. 1992, Astrophysics of neutron stars, Springer Verlag

Lyne A.G. \& Lorimer D.R., 1994, Nature, 369, 127

Madau P. \& Blaes O., 1994, ApJ, 423, 748

Miller M.C., 1992, MNRAS, 255, 129

Morrison R. \& McCammon D., 1983, ApJ, 270, 119

Narayan R. \& Ostriker J.P., 1990, ApJ, 352, 222

Nelson R.W., Wang J.C.L., Salpeter E.E. \& Wasserman I., 1995, ApJ, 438, L99

Novikov I.D. \& Thorne K.S., 1973, in "Black Holes", eds. C. DeWitt \& B.S. DeWitt (New York: Gordon \& Break), 343

Ostriker J.P., Rees M.J. \& Silk J., 1970, Astrophys. Letters, 6, 179 
Paczyński B., 1990, ApJ, 348, 485

Paresce F., 1984, AJ, 89, 1022

Pfeffermann E. et al., 1986, SPIE, 733, 519

Postman M. et al., 1995, in preparation

Shemi A., 1995, MNRAS, 275, L7

Shibanov Yu.A., Zavlin V.E., Pavlov G.G. \& Ventura J., 1992, A\&A, 266, 313

Stocke J.T., Wang Q.D., Perlman E.S., Donahue M. \& Schachter J., 1995, AJ, 109, 1199

Treves A. \& Colpi M., 1991, A\&A, 241, 107

Trümper J., 1983, Adv. Space Res., 2, 241

Walter F.M., Wolk S.J. \& Neuhäuser R., 1996, Nature, 379, 233

Welsh B.Y., Craig N., Vedder P.W. \& Vallerga J.V., 1994, ApJ, 437, 638

Wielen R., 1977, A\&A, 60, 263

Zampieri L., Turolla R., Zane S. \& Treves A., 1995, ApJ, 439, 849

Zane S., Turolla R., Zampieri L., Colpi M. \& Treves A., 1995a, ApJ, 451, 739

Zane S., Zampieri L., Turolla R. \& Treves A., 1995b, A\&A, in press

Zimmermann H.U. et al., 1994, MPE Report 257 


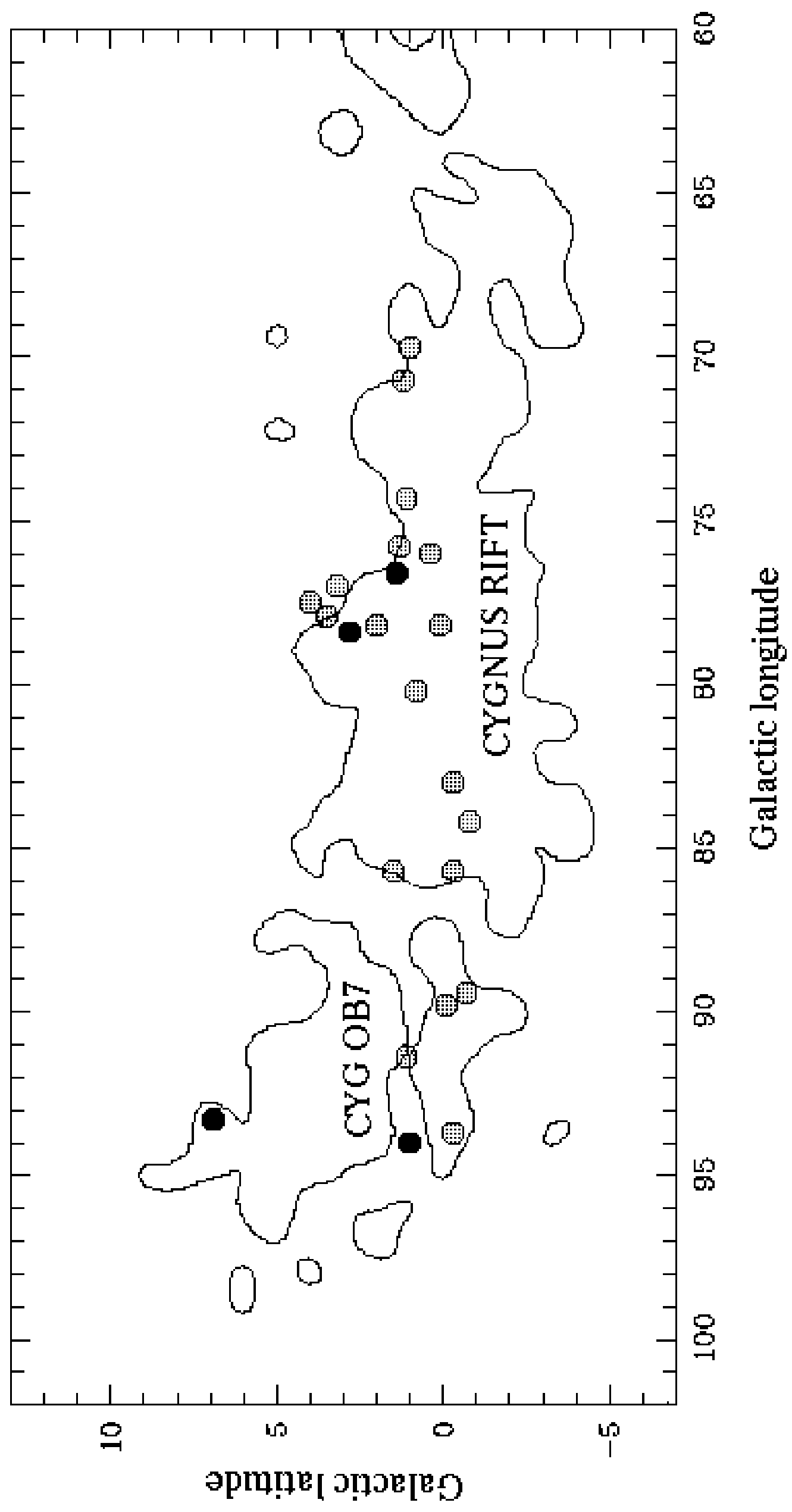




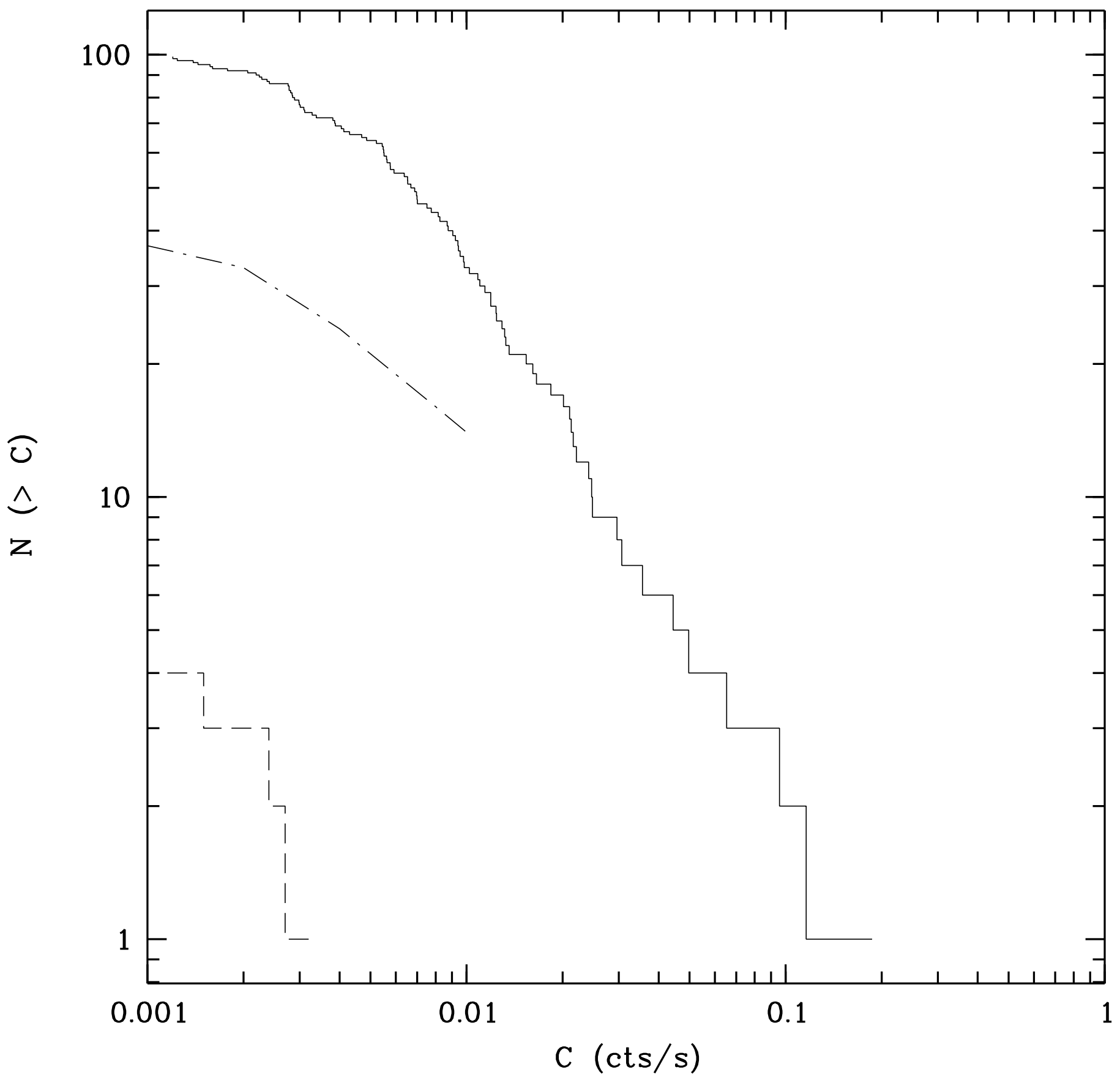




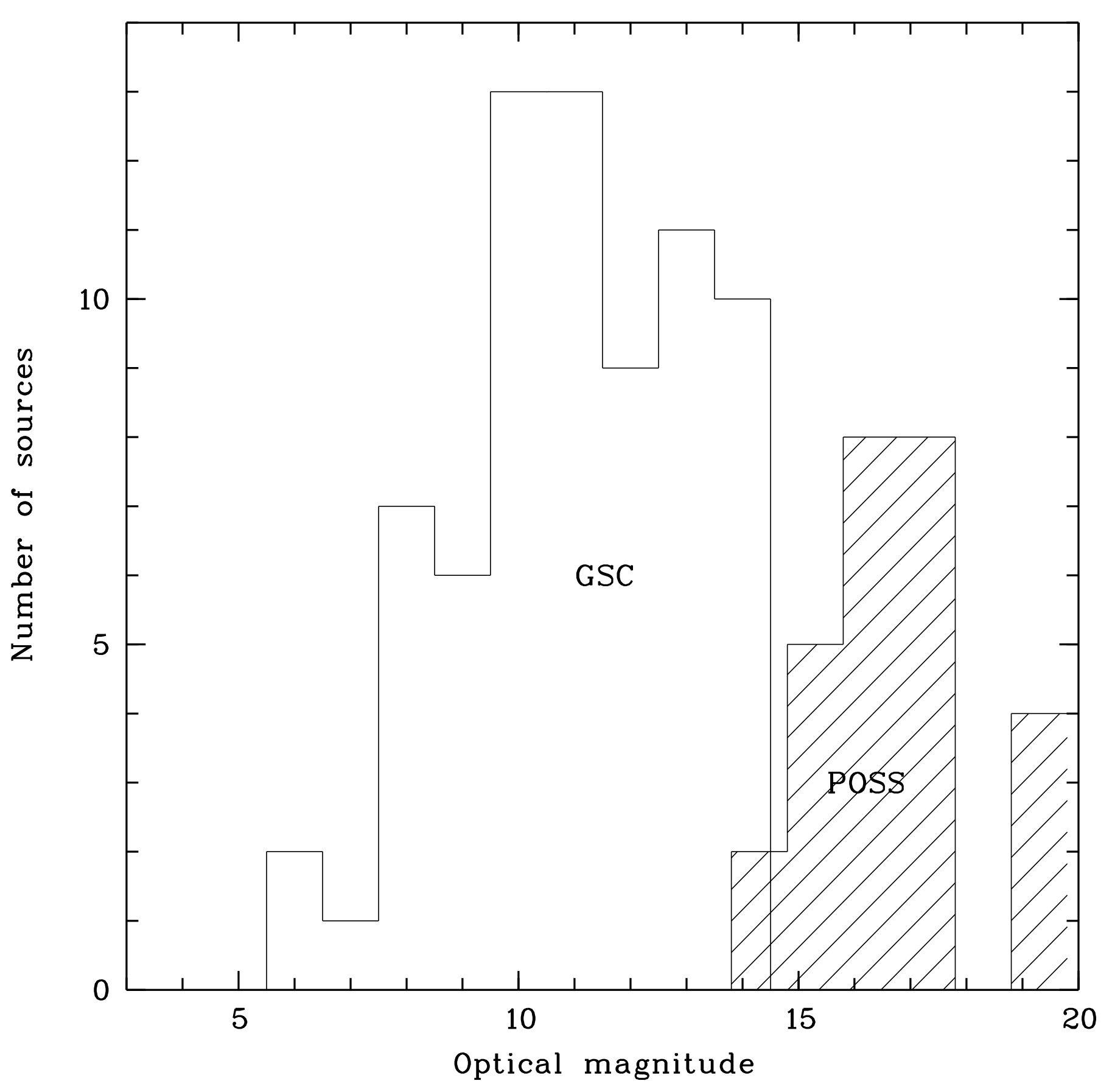

